\title{
Optical Coherence Tomography and Fibrous Cap Characterization
}

\author{
Daniel Chamié • Zhao Wang • Hiram Bezerra • \\ Andrew M. Rollins • Marco A. Costa
}

Published online: 12 May 2011

(C) Springer Science+Business Media, LLC 2011

\begin{abstract}
The pathophysiology of acute coronary syndromes has long been associated with atherosclerotic plaque rupture. Inflammation, thinning, and disruption of the fibrous cap have been implicated with the final processes leading to plaque rupture, but confirmation of these mechanisms of coronary thrombosis in humans has been hampered by the lack of imaging methods with sufficient resolution to resolve fibrous cap characterization and thickness in vivo. Intravascular optical coherence tomography (OCT) provides images with micron-level axial and lateral resolution, enabling detailed visualization of micro-structural changes of the arterial wall. The present article provides an overview of the potential role of OCT in identifying and characterizing fibrous cap morphology, thickness, and inflammation in human coronary plaques.
\end{abstract}

Keywords Optical coherence tomography · OCT · Fibrous cap · Thin-cap fibroatheroma

\section{Introduction}

Acute coronary thrombosis has been shown to result from disruption of the protective fibrous cap and exposure of a

D. Chamié $\cdot$ H. Bezerra $\cdot$ M. A. Costa $(\bowtie)$

Division of Cardiology, Harrington-McLaughlin Heart and Vascular Institute, University Hospitals Case Medical Center, Case Western Reserve School of Medicine,

11100 Euclid Avenue, Lakeside 3001,

Cleveland, OH 44106, USA

e-mail: marco.costa@uhhospitals.org

Z. Wang $\cdot$ A. M. Rollins

Department of Biomedical Engineering,

Case Western Reserve University,

Cleveland, $\mathrm{OH}$, USA prothrombotic necrotic core to the blood stream $[1,2]$. Atherosclerotic plaques most likely to rupture have been characterized as those containing a large lipid necrotic core covered by a thin fibrous cap, which is infiltrated by macrophages $[3,4]$. The daunting task of identifying the socalled "vulnerable plaques" before symptoms arise and prevent subsequent myocardial infarction (MI) or sudden death has stirred the minds of many clinicians and scientists. However, it has become clear that our understanding of the pathophysiology of acute coronary syndrome (ACS) remains incomplete.

Thickness of the fibrous cap has been suggested as a marker of plaque rupture. Further understanding of the role of fibrous cap as a risk factor of ACS demands highresolution in vivo imaging in humans. The recent introduction of intravascular optical coherence tomography (OCT), a new imaging technique that uses near-infrared light to provide high-resolution $(10-15 \mu \mathrm{m})$ imaging, makes it possible to visualize the arterial wall microenvironment [5]. This article provides an overview of the potential role of intravascular OCT in the characterization of fibrous plaque morphology, with emphasis on validation studies and current clinical applications. A brief review of the basic principles of this novel imaging modality and future perspectives are also discussed.

\section{The Fibrous Cap}

The fibrous cap has been defined as a distinct layer of connective tissue covering the lipid core of an atherosclerotic plaque, and consists purely of smooth muscle cells in a collagenous-proteoglycan matrix, with varying degrees of infiltration by macrophages and lymphocytes [4]. Rupture of the fibrous cap exposes prothrombotic material, which 
triggers platelet activation, aggregation, thrombin formation, and ultimately thrombosis. Disruption of the fibrous cap has been identified in $60 \%$ to $75 \%$ of pathology specimens from patients dying of acute coronary thrombosis, followed by plaque erosion $(25 \%-30 \%)$, and calcified nodule $(2 \%-7 \%)[4,6,7]$. Transition of "stable plaques" to plaque rupture has been characterized by the presence of endothelial denudation with superficial platelet aggregation, large lipid necrotic core, active inflammation (monocyte/ macrophage infiltration), thinning of the fibrous cap, and intraplaque hemorrhage [8]. The exact mechanism of fibrous cap thinning has not been fully elucidated, but necropsy studies found ruptured plaques to have fibrous caps $<65 \mu \mathrm{m}$ in thickness [9]. The data come from an ex vivo morphometric assessment of 41 ruptured plaques, in which the mean cap thickness was $23 \pm 19 \mu \mathrm{m}$ and $95 \%$ of the caps measured less than $64 \mu \mathrm{m}$ [9]. The data are plagued by methodological flaws in measuring the fibrous cap, a three-dimensional (3-D) structure, in single crosssections and by potential histology preparation artifacts, which inherently leads to shrinkage of the tissue. Importantly, cause-effect relationships cannot be derived from the data. Nevertheless, the thin-cap fibroatheroma (TCFA) has been considered the non-thrombosed lesion that mostly resembles the ruptured plaque, and has been characterized by a necrotic core with an overlying thin fibrous cap $(<65 \mu \mathrm{m})$ infiltrated by macrophages $(>25$ cells per $0.3 \mathrm{~mm})$ containing rare smooth muscle cells $[3,4,7]$.

\section{Current Imaging Modalities}

\section{Angiography}

Coronary angiography has long been considered the "gold standard" method to assess the severity of luminal narrowing, serving as a diagnostic and decision-making tool in the catheterization laboratory. It allows for the assessment of lumen silhouette, but does not provide information regarding the arterial wall or plaque. Most acute coronary occlusions occur in "angiographically normal" segments, and only a minority occur at sites of severe stenosis [10].

\section{Intravascular Ultrasound}

Intravascular ultrasound (IVUS) provides real-time tomographic cross-sectional images of the coronary vessel wall, providing valuable insights into quantification and composition of coronary plaques, such as eccentricity, hypoechogenicity, and positive remodeling. Its limited axial resolution $(\sim 200 \mu \mathrm{m})$ precludes assessment of the fibrous caps [11].
Radiofrequency IVUS Data Analysis (Virtual Histology)

Autoregressive spectral analysis of IVUS backscattered radiofrequency signal allows characterization of different plaque components. This technique has been coined virtual histology IVUS (VH-IVUS), although its correlation with true histology is only marginal [12]. Plaque components are assigned a color-coded scheme according to tissue ultrasound signal properties into four different types of plaque components [13]. The VH-IVUS proposed definition of "TCFA" is a scientific misnomer, as neither the fibrous cap nor its thickness are considered due to limited axial resolution of the method [14].

\section{Optical Coherence Tomography}

\section{Basic Principles}

Intravascular OCT is a catheter-based image modality that produces high-resolution cross-sectional imaging of biological tissues by measuring the magnitude and "echo time delay" of backscattered light, instead of ultrasound. Since the speed of light $\left(3 \times 10^{8} \mathrm{~m} / \mathrm{s}\right)$ is much faster than that of sound $(1,500 \mathrm{~m} / \mathrm{s})$, an interferometer is required to measure the backscattered signal. The interferometer uses a fiberoptic coupler similar to a beam splitter, which directs half of the beam to the measurement arm (tissue) and the other half to the reference arm. The light source used by intravascular OCT has a near-infrared spectrum, with wavelengths ranging from 1,250 to $1,350 \mathrm{~nm}$. The axial resolution of current OCT systems ranges from 10 to $15 \mu \mathrm{m}$ [5], which allows detailed visualization of biological structures at a micro-scale level [5]. Cross-sectional images are created based on a series of multiple axial scans (A-scans) at different transverse positions in order to generate a two-dimensional dataset (B-scan). Newer OCT systems use a fixed mirror with a variable frequency light source, or "swept-laser," to produce known echo delays. This allows the system to acquire 100 to 200 frames/s with a pullback speed of up to $40 \mathrm{~mm} / \mathrm{s}$, having the potential to scan $4-6 \mathrm{~cm}$ length of a coronary artery in less than $5 \mathrm{~s}$. Importantly, red blood cells cause significant attenuation of the light signal, requiring blood to be completely displaced prior to image acquisition. The high pullback speed eliminates the need for balloon occlusion to clear the blood, which is now achieved by a contrast bolus injection [5].

\section{OCT and Fibrous Cap: Identification}

Light is absorbed by lipids, leading to a region of low or no signal, casting the vascular wall beyond in shadow [5]. Identification of the lipid-necrotic core represents a funda- 
mental step toward identifying the fibrous cap. Ability of OCT to detect lipid-rich plaques has been demonstrated ex vivo [15]. Three hundred and fifty-seven diseased arterial segments were examined by histology and OCT image. On OCT, fibrous tissue appears as homogenous, signal-rich regions; fibrocalcific or calcific tissue appear as welldelineated, signal-poor regions with sharp borders; and lipids are depicted as signal-poor regions with diffuse borders [15]. OCT showed $94 \%$ sensitivity and $92 \%$ specificity to detect lipid-rich plaques. Furthermore, others found that lipid plaques were identified more frequently by OCT compared with IVUS [16]. The interface between the fibrous tissue and lipidic necrotic core produces a high superficial backscattering signal. The combination of a signal-rich band overlying a signal-poor region produces a unique, distinct signature, which can be easily identified on OCT images (Fig. 1).

\section{OCT and Fibrous Cap: Measurement}

A preclinical validation study assessed the feasibility of OCT to determine fibrous cap thickness using histology as the "gold standard" in 35 lipid-rich plaques from 102 coronary arterial segments of 38 human cadavers [17]. Fibrous cap was defined by OCT as the minimum axial distance from the coronary lumen to the inner border of the lipid pool, and its thickness was measured at the thinnest portion in each plaque. The thickness of the fibrous cap ranged from 10 to $450 \mu \mathrm{m}$ (mean $138 \mu \mathrm{m}$ ) by histology and 10 to $430 \mu \mathrm{m}$ (mean $163 \mu \mathrm{m}$ ) by OCT. Good correlation $(r=0.90, P<0.001)$ and agreement (mean difference, $-24 \pm 44 \mu \mathrm{m}$ ) were observed between the OCT and histologic measurements, with surprisingly low intraobserver $(13 \pm 41 \mu \mathrm{m})$ and interobserver $(20 \pm 59 \mu \mathrm{m})$ variability reported [17].

Culprit lesions of 30 patients with MI were examined with OCT, IVUS, and angioscopy. OCT was the only method able to estimate the fibrous cap thickness at $49 \pm$ $21 \mu \mathrm{m}$, using similar methodology as described above. In addition, the study revealed, for the first time, the ability to detect fibrous cap erosion in vivo using OCT $(23 \%)$, but not by IVUS [18].

Recent OCT studies have revealed that the commonly used threshold of $65 \mu \mathrm{m}$ to define a thin fibrous cap associated with plaque rupture [9] may not be appropriate
Fig. 1 Representative cross-sectional images of coronary atherosclerosis by OCT. a, A mild concentric fibrous plaque, characterized by homogenous, signal-rich regions. Lipidic plaques (b, c, and d) are characterized by superficial signal-rich region followed by a drop in signal, as a result of high backscattering and attenuation (asterisks). The fibrous cap can be identified as a homogeneous signal-rich band overlying the lipid-rich core. b, A lipid-rich plaque with thick cap (cap thickness at the thinnest part measured $178 \mu \mathrm{m}$ ). c, A thin-cap fibroatheroma, defined by a thin-cap $(46 \mu \mathrm{m}$ at its thinnest part) overlying a lipid-rich plaque. d, Rupture of the fibrous cap overlying a lipid-rich plaque can be observed
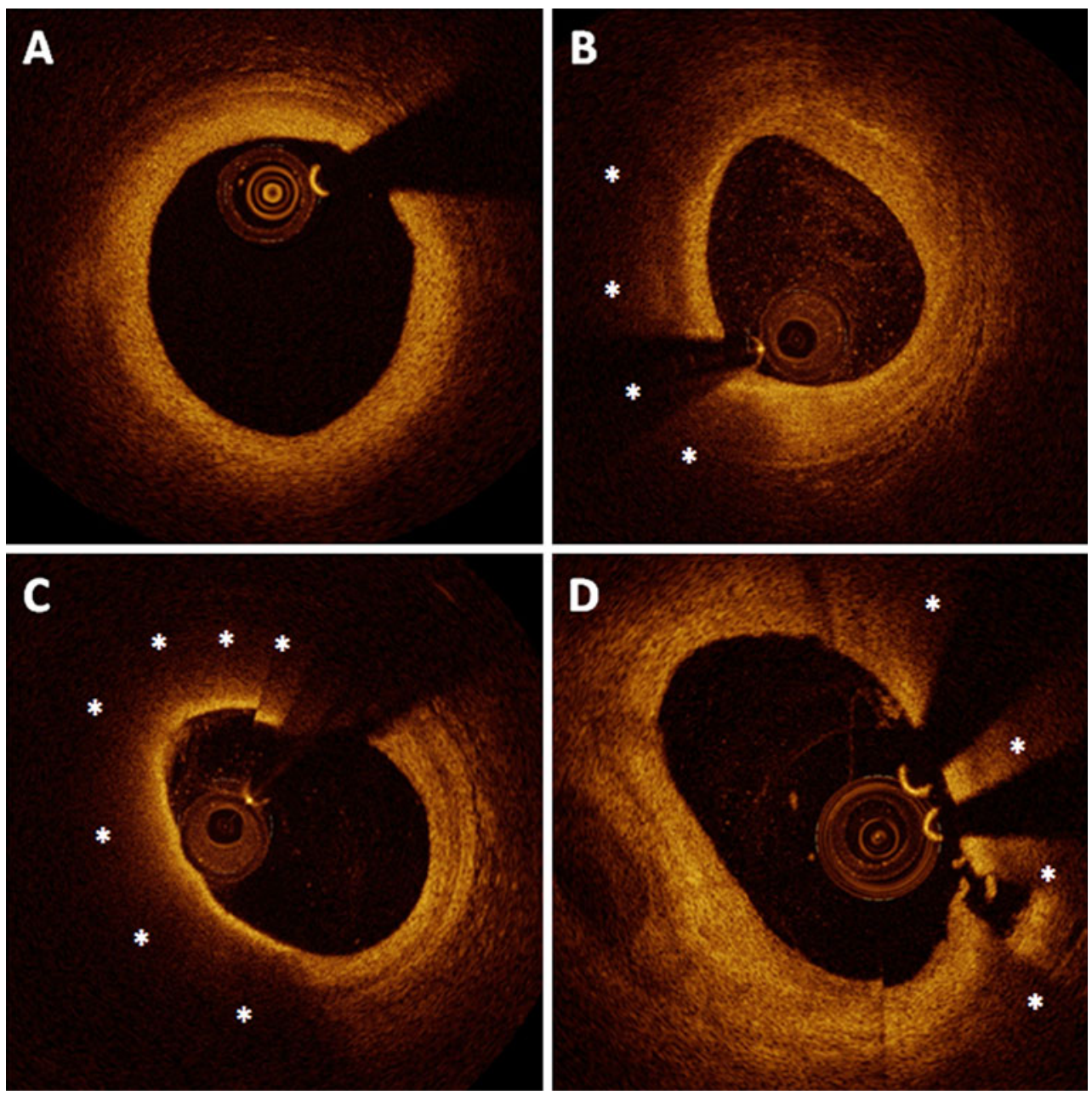
in nonruptured plaques. In vivo OCT examinations have shown that fibrous cap thickness varies widely between ruptured and nonruptured plaques. In a recent clinical study with 266 patients, the median thinnest cap thickness was $54 \mu \mathrm{m}$ in ruptured plaques, compared with $80 \mu \mathrm{m}$ in nonruptured plaques. The median, most representative cap thickness was also significantly thinner in the ruptured plaques (116 vs $182 \mu \mathrm{m}, P=0.01$ ). In $95 \%$ of the ruptured plaques, the thinnest cap thickness and most representative cap thickness were $<80$ and $<188 \mu \mathrm{m}$, respectively. Of note, the best cutoff for predicting rupture was $151 \mu \mathrm{m}$ (OR: 35.6, 95\% CI $=15.0-84.3, P<0.001$ ) for most representative cap thickness [19••].

Fibrous cap thickness has also been observed to vary according to clinical presentation. Patients with ST elevation MI (STEMI) showed significantly lower median mean values of the minimum thickness of the fibrous cap $(47 \mu \mathrm{m})$ in comparison to non-ST elevation MI (NSTEMI) $(53.8 \mu \mathrm{m})$ and stable angina $(102.6 \mu \mathrm{m})$ patients $(P=$ 0.034 for all comparisons) [20]. Interestingly, fibrous cap thickness has also been shown to be thinner in patients presenting with resting angina $(50 \mu \mathrm{m})$ in comparison to patients presenting with angina triggered by exertion $(90 \mu \mathrm{m})[21 \bullet \bullet]$. In another report comparing culprit lesion morphologies between STEMI and NSTEMI patients by OCT, fibrous cap thickness was significantly thinner $(55 \pm$ $20 \mu \mathrm{m}$ vs $109 \pm 55 \mu \mathrm{m}, P<0.001)$ in STEMI patients. Importantly, a "proximal-type" rupture of the fibrous cap was more often seen in STEMI ( $46 \%$ vs $17 \%, P=0.039$ ), while "distal-type" aperture was more often observed in NSTEMI ( $11 \%$ vs $35 \%, P=0.048)$, suggesting that longitudinal morphology and location of cap rupture, as visualized by OCT, may contribute to the variable clinical presentation of plaque rupture [22•]. Collectively, these early OCT studies revealed unprecedented features of the fibrous cap and establish a solid foundation for future research. However, methodological refinements are still required to advance our understanding of the pathophysiology of ACS and plaque rupture. The retrospective nature of these studies, similar to necropsy investigations, precludes a better assessment of the cause-effect relationship between fibrous cap thickness and plaque rupture. For instance, it is impossible to determine cap thickness at the location of the rupture, limiting assessment to the adjacent segments of the cap.

Current methodology for determining fibrous cap thickness is derived from histology and relies on manual measurement at a single point or by averaging multiple measurements $[17,18],[19 \bullet \bullet],[20],[22 \bullet],[23-25]$ at locations presumed, by visual assessment, to be the thinnest region in a cross-sectional image. However, the fibrous cap is a dynamic 3-D structure subjected to a variety of mechanical, biological, chemical, and inflammatory factors and its thickness varies considerably along the plaque. Performing random measures in a single or few crosssections will likely not capture the complex 3-D nature of the fibrous cap.

\section{OCT and Fibrous Cap: 3-D Assessment}

Our group is working on establishing a 3-D display of the fibrous cap that allows volumetric quantification of thickness to overcome these limitations. The ultimate goal is to have automated identification of lipid plaques with real-time, comprehensive, and accurate determination of the morphological features of the fibrous cap, with minimal requirement for observer interference. Briefly, after determining the region of interest (lipidrich plaque), a computer program automatically segments the entire extent of the fibrous cap. Cap thickness in every locus is then quantified with respect to the centroid of the lumen. A color classification scheme is constructed in a 3-D-rendered image, mapping the entire extent of the fibrous cap in different regions according to its thickness in red $(<65 \mu \mathrm{m})$, green $(65-150 \mu \mathrm{m})$, or blue $(>150 \mu \mathrm{m})$ (Fig. 2). Hence, minimal, maximal, and mean values of the fibrous cap thickness can be determined along with the quantification of area and volume of the whole cap. The 3-D display enables a comprehensive visualization of the longitudinal and circumferential distribution of the fibrous cap, and identification of "hotspots" of thin regions.

\section{OCT and Fibrous Cap: Inflammation}

The central role of active inflammation in the pathophysiology underlying arterial thrombosis has been well described [26, 27]. Macrophage infiltration of the fibrous cap of atherosclerotic plaques is thought to weaken the structural integrity of the cap, predisposing plaques to rupture $[28,29]$. Macrophages/foam cells in atherosclerotic plaques are relatively large $(20-30 \mu \mathrm{m})$ and may contain multiple intracellular, lipid-rich phagolysosomes. It has been postulated that as a function of their size and the refractive index difference between the phagolysosomes and the surrounding intracellular fluid, macrophages may have a higher OCT signal than the surrounding tissue [30, 31]. A high positive correlation between OCT and histological measurements of fibrous cap macrophage density $(r=0.84, P<0.0001)$, and a negative correlation between OCT and histological measurements of smooth muscle actin density $(r=-0.56, P<0.005)$ have been demonstrated in an ex vivo experiment [30]. Eighty-four percent of the OCT images depicted signs of macrophage infiltration at the interface between the cap and the lipid necrotic core of "vulnerable plaques" [32]. 

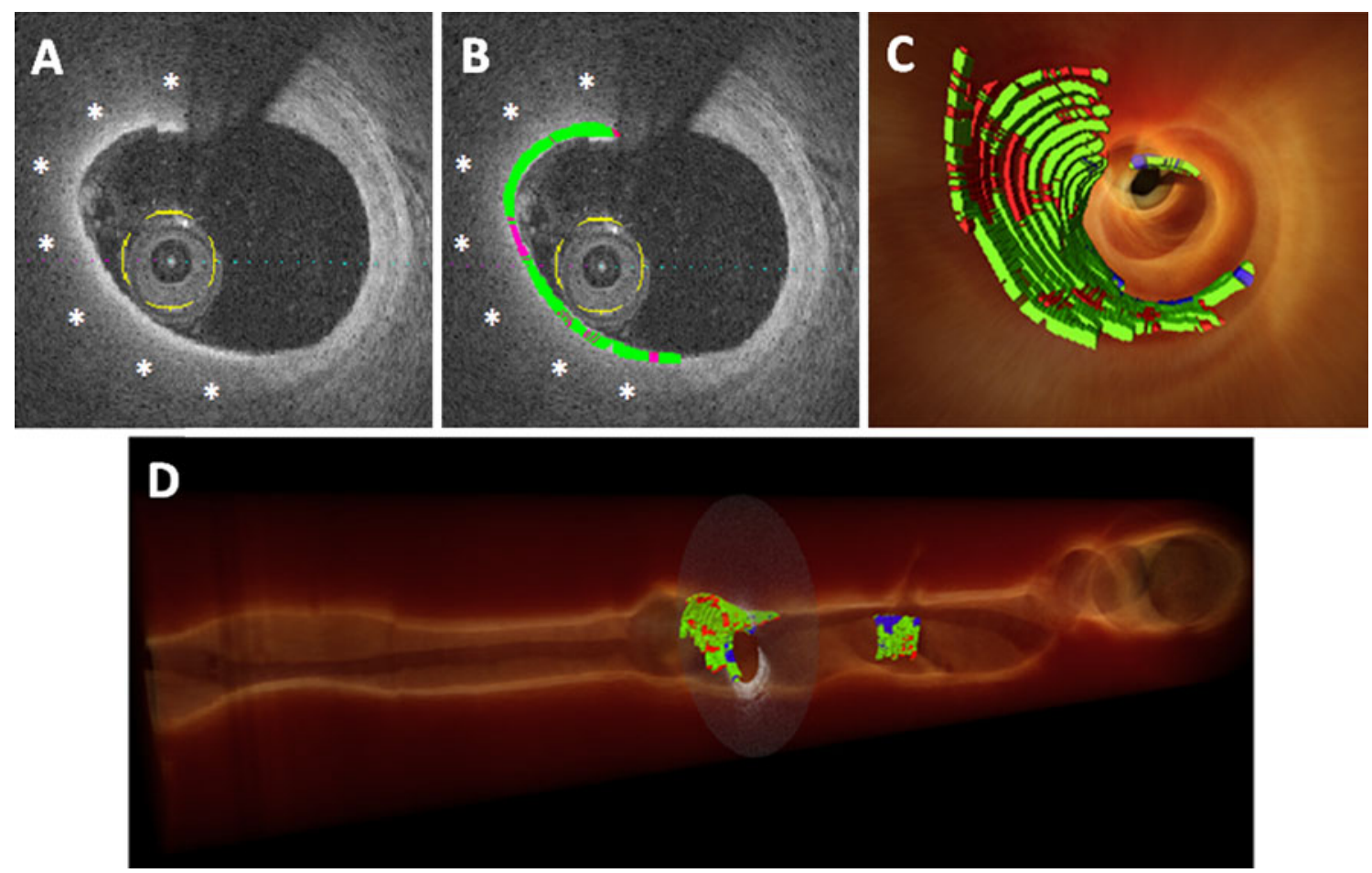

Fig. 2 Volumetric method for quantification of fibrous caps. a, A two-dimensional cross-section image depicting a lipid-rich plaque (asterisks). b, After segmenting the entire fibrous cap extension, a color classification scheme is constructed, mapping the whole fibrous cap according to the regional thickness in red $(<65 \mu \mathrm{m})$, green $(65-$ $150 \mu \mathrm{m})$, or blue $(>150 \mu \mathrm{m})$. The fibrous cap is segmented in all frames containing a lipid-rich plaque, allowing for real-time volumetric assessment. c, 3-D rendering reconstruction with fly-through of the coronary vessel, demonstrating the thickness quantification mapping

ACS patients had significantly greater macrophage density based on OCT parameters, both for fibrous and lipid-rich plaques ( $P=0.025$ and $P=0.002$, respectively), than patients with stable angina [33]. In addition, sites of plaque rupture demonstrated a greater macrophage density than nonruptured sites $(6.95 \% \pm 1.60 \%$ vs $5.29 \% \pm 1.17 \%$, $P=0.002$ ). Good correlation between macrophage density in the fibrous cap and white blood cell count $(r=0.483, P<$ 0.001 ) has also been described. Both parameters were associated with lipid-rich plaque and correlated inversely with fibrous cap thickness $(r=-0.547$ for macrophage density and -0.423 for white blood cell count, $P<0.015$ ) [34]. A recent small study with 25 ACS patients demonstrated that baseline systemic levels of myeloperoxidase were significantly higher in patients with eroded plaques than in patients with ruptured plaques (median, 2500 [IQR: $1,415-2,920] \mathrm{ng} / \mathrm{mL}$ vs 707 [312-943] ng/mL, $P=0.001$ ) as determined by OCT, although high-sensitivity $\mathrm{C}$-reactive protein (hs-CRP) levels did not differ significantly (median, $11.3[1.3-28.5] \mathrm{mg} / \mathrm{L}$ vs $3.9[1.3-17.8] \mathrm{mg} / \mathrm{L}, P=0.76)$ [35•]. Systemic levels of hs-CRP have also been found to be significantly higher in ACS patients with $\geq 1$ TCFA of the whole cap. d, Longitudinal view, allowing a broad visualization of longitudinal and circumferential distribution of the thickness of the fibrous cap. The thresholds of fibrous cap thickness used in this representative example were selected according to the current criteria derived from pathology. However, this novel method allows for quantification of fibrous caps to move from a binary question to a continuous variable, changing the concept of finding a single threshold to predict plaque rupture into a more comprehensive assessment of this complex and dynamic structure

detected by OCT in the target vessel, in comparison to nonTCFA ACS patients [24]. These observations, although retrospective in nature and preliminary, suggest a preferential spatial distribution of macrophages in culprit lesions within the same patient, even in the setting of a generalized inflammatory process. Whether inflammation preceded the clinical event (ie, plaque rupture) or it was a consequence and therefore a marker of disease remains to be demonstrated. Nevertheless, these findings indicate a potential clinical role of OCT in identifying inflammation on the surface of lipid-rich plaques, which could become a new surrogate of plaque vulnerability.

\section{OCT and Fibrous Cap: Therapeutic Implications}

Several IVUS-based studies have been conducted over the last decade to evaluate the effects of different lipid-lowering drugs on atheroma size [36, 37]. Although the efficacy of lowering low-density lipoprotein cholesterol with inhibitors of hydroxymethylglutaryl-coenzyme A reductase (statins) is unequivocal, the change in atheroma size promoted by statins has not been consistent across all IVUS studies [36]. 
The commonly used parameters of changes of total atheroma volume or percent change of atheroma volume may be affected by vessel size and remodeling, and the role of these quantitative variables on the pathophysiology of ACS remains questionable.

OCT has been recently used to assess the effects of statins on coronary fibrous cap thickness in ACS patients. Serial OCT examinations at baseline and 9-month followup revealed that the fibrous cap thickness significantly increased in both the statin-treated group $(P<0.01)$ and control group $(P<0.01)$ over time [38]. However, the degree of increase was significantly greater in the statintreated group $(188 \% \pm 64 \%$ vs $117 \% \pm 39 \%, P<0.01)$ [39••]. Another study showed that patients previously in use of statins had a significantly lower frequency of ruptured culprit plaques than patients not taking statins ( $8 \%$ vs $36 \%$, $P=0.03$ ), with a trend toward an increase in the fibrous cap thickness (78 vs $49 \mu \mathrm{m}, P=0.07$ ).

Although systemic therapies appear more attractive for "stabilization" of vulnerable plaques, an interventional approach applied locally to asymptomatic vulnerable plaques has been proposed [6, 40]. In the Providing Regional Observations to Study Predictors of Events in the Coronary Tree (PROSPECT) trial, the natural history of coronary atherosclerosis was investigated using multimodality intravascular imaging of the three major coronary arteries, with patients under optimal medical therapy, including statins. A VH-IVUS-derived TCFA with a minimum lumen area $\leq 4.0 \mathrm{~mm}^{2}$ and a large plaque burden $(\geq 70 \%$ ) had a likelihood of causing an ischemic event within 3 years of $17.2 \%$. However, the anticipated high frequency of acute thrombotic cardiovascular events did not occur, with only $1 \%$ rate of MI and no deaths directly attributed to nonculprit vessels over 3-year follow-up. These results suggest that nonculprit, yet obstructive coronary plaques, are most likely to be associated with increasing symptoms rather than thrombotic acute events, with $8.5 \%$ of patients presenting with worsening angina and $3.3 \%$ with unstable angina [41]. Studies using OCT and more refined characterization of the plaque, fibrous cap, and inflammatory process are still pending.

\section{Future Perspectives}

Advances in OCT technology may further increase the anatomical and functional assessment of the fibrous cap. Interstitial collagen and smooth muscle cells are closely involved in the biomechanical strength of the fibrous cap, conferring stability. Polarization-sensitive OCT has proven capable of measuring birefringence (a material property that is elevated in tissues such as collagen and smooth muscle cells). An ex vivo study demonstrated that polarization- sensitive OCT enables the measurement of birefringence in plaques and in fibrous caps of necrotic core fibroatheromas, with potential to evaluate collagen content, collagen fiber thickness, and smooth muscle cell density [42]. Furthermore, nanomedicine is a fast evolving field and development of nanoparticles targeted against specific antibodies might allow for an easy way to detect and quantify macrophage in the fibrous cap by OCT.

\section{Conclusions}

Fibrous cap integrity is an important prerequisite for plaque stability. TCFA is identified, on the basis of several pathological studies, as the precursor for plaque rupture, the most common cause of acute coronary thrombosis and ACS. Evaluation of fibrous cap thickness is critical for characterization of TCFA. Until recently, accurate assessment of the fibrous cap in vivo was not possible due to the lack of available imaging modalities with high resolution. The recently introduced intravascular OCT with micrometer resolution has proven to be a feasible and reproducible method to resolve the fibrous cap structural aspects. OCT may fill an important gap in our knowledge regarding the true natural history of atherosclerotic plaques, with potential to increase current in vivo diagnostic capacity of proneto-rupture plaques, and assist in the development of specific preventive and therapeutic approaches.

Disclosure D. Chamié: none; Z. Wang: none; H. Bezerra: honoraria from St. Jude Medical; A. M. Rollins: none; M. A. Costa: honoraria from St. Jude Medical.

\section{References}

Papers of particular interest, published recently, have been highlighted as:

- Of importance

- Of major importance

1. Falk E. Plaque rupture with severe pre-existing stenosis precipitating coronary thrombosis. Characteristics of coronary atherosclerotic plaques underlying fatal occlusive thrombi. Br Heart J. 1983;50(2):127-34.

2. Davies MJ, Thomas A. Thrombosis and acute coronary-artery lesions in sudden cardiac ischemic death. N Engl J Med. 1984;310 (18): $1137-40$.

3. Virmani R, Burke AP, Farb A, Kolodgie FD. Pathology of the unstable plaque. Prog Cardiovasc Dis. 2002;44(5):349-56.

4. Virmani R, Kolodgie FD, Burke AP, et al. Lessons from sudden coronary death: a comprehensive morphological classification scheme for atherosclerotic lesions. Arterioscler Thromb Vasc Biol. 2000;20(5):1262-75. 
5. Bezerra HG, Costa MA, Guagliumi G, et al. Intracoronary optical coherence tomography: a comprehensive review clinical and research applications. JACC Cardiovasc Interv. 2009;2(11):103546.

6. Kolodgie FD, Virmani R, Burke AP, et al. Pathologic assessment of the vulnerable human coronary plaque. Heart. 2004;90 (12):1385-91.

7. Virmani R, Burke AP, Farb A, Kolodgie FD. Pathology of the vulnerable plaque. J Am Coll Cardiol. 2006;47(8 Suppl):C13-18.

8. Kolodgie FD, Gold HK, Burke AP, et al. Intraplaque hemorrhage and progression of coronary atheroma. N Engl J Med. 2003;349 (24):2316-25.

9. Burke AP, Farb A, Malcom GT, et al. Coronary risk factors and plaque morphology in men with coronary disease who died suddenly. N Engl J Med. 1997;336(18):1276-82.

10. Little WC, Constantinescu M, Applegate RJ, et al. Can coronary angiography predict the site of a subsequent myocardial infarction in patients with mild-to-moderate coronary artery disease? Circulation. 1988;78(5 Pt 1):1157-66.

11. Mintz GS, Nissen SE, Anderson WD, et al. American College of Cardiology Clinical Expert Consensus Document on Standards for Acquisition, Measurement and Reporting of Intravascular Ultrasound Studies (IVUS). A report of the American College of Cardiology Task Force on Clinical Expert Consensus Documents. J Am Coll Cardiol. 2001;37(5):1478-92.

12. Thim T, Hagensen MK, Wallace-Bradley D, et al. Unreliable assessment of necrotic core by virtual histology intravascular ultrasound in porcine coronary artery disease. Circ Cardiovasc Imaging. 2010;3(4):384-91.

13. Nair A, Margolis MP, Kuban BD, Vince DG. Automated coronary plaque characterisation with intravascular ultrasound backscatter: ex vivo validation. EuroIntervention. 2007;3(1):113-20.

14. Rodriguez-Granillo GA, Garcia-Garcia HM. Mc Fadden EP, et al. In vivo intravascular ultrasound-derived thin-cap fibroatheroma detection using ultrasound radiofrequency data analysis. J Am Coll Cardiol. 2005;46(11):2038-42.

15. Yabushita H, Bouma BE, Houser SL, et al. Characterization of human atherosclerosis by optical coherence tomography. Circulation. 2002;106(13):1640-5.

16. Jang IK, Bouma BE, Kang DH, et al. Visualization of coronary atherosclerotic plaques in patients using optical coherence tomography: comparison with intravascular ultrasound. J Am Coll Cardiol. 2002;39(4):604-9.

17. Kume T, Akasaka T, Kawamoto T. Measurement of the thickness of the fibrous cap by optical coherence tomography. Am Heart J. 2006;152(4):755. e751-754.

18. Kubo T, Imanishi $\mathrm{T}$, Takarada $\mathrm{S}$, et al. Assessment of culprit lesion morphology in acute myocardial infarction: ability of optical coherence tomography compared with intravascular ultrasound and coronary angioscopy. J Am Coll Cardiol. 2007;50(10):933-9.

19. • Yonetsu T, Kakuta T, Lee T, et al. In vivo critical fibrous cap thickness for rupture-prone coronary plaques assessed by optical coherence tomography. Eur Heart J. 2011.Important study challenging the current paradigm derived from pathology studies by determining the in vivo thresholds of fibrous cap thickness for predicting plaque rupture.

20. Jang IK, Tearney GJ, MacNeill B, et al. In vivo characterization of coronary atherosclerotic plaque by use of optical coherence tomography. Circulation. 2005;111(12):1551-5.

21. •Tanaka A, Imanishi $\mathrm{T}$, Kitabata $\mathrm{H}$, et al. Morphology of exertion-triggered plaque rupture in patients with acute coronary syndrome: an optical coherence tomography study. Circulation. 2008;118(23):2368-2373.Fibrous cap rupture can occur at sites with different thickness depending on the trigger stimulus. This observation challenges the current practice of just searching for the thinnest part of the cap, and praises the need for a more comprehensive and volumetric analysis of this complex structure.

22. -Ino Y, Kubo T, Tanaka A, et al. Difference of Culprit Lesion Morphologies Between ST-Segment Elevation Myocardial Infarction and Non-ST-Segment Elevation Acute Coronary Syndrome An Optical Coherence Tomography Study. JACC Cardiovasc Interv. 2011;4(1):76-82.This study highlights the importance of studying the morphology of fibrous caps. The authors suggest that beyond the determination of fibrous cap dimensions, careful analysis of its longitudinal morphology can relate to clinical presentation in ACS patients.

23. Rathore S, Terashima M, Matsuo H, et al. In-vivo detection of the frequency and distribution of thin-cap fibroatheroma and ruptured plaques in patients with coronary artery disease: an optical coherence tomographic study. Coron Artery Dis. 2011;22(2):6472.

24. Tanaka A, Imanishi $\mathrm{T}$, Kitabata $\mathrm{H}$, et al. Distribution and frequency of thin-capped fibroatheromas and ruptured plaques in the entire culprit coronary artery in patients with acute coronary syndrome as determined by optical coherence tomography. Am J Cardiol. 2008;102(8):975-9.

25. Sawada T, Shite J, Garcia-Garcia HM, et al. Feasibility of combined use of intravascular ultrasound radiofrequency data analysis and optical coherence tomography for detecting thin-cap fibroatheroma. Eur Heart J. 2008;29(9):1136-46.

26. Libby P, Simon DI. Inflammation and thrombosis: the clot thickens. Circulation. 2001;103(13):1718-20.

27. Libby P. Current concepts of the pathogenesis of the acute coronary syndromes. Circulation. 2001;104(3):365-72.

28. Lendon CL, Davies MJ, Born GV, Richardson PD. Atherosclerotic plaque caps are locally weakened when macrophages density is increased. Atherosclerosis. 1991;87(1):87-90.

29. Moreno PR, Falk E, Palacios IF, et al. Macrophage infiltration in acute coronary syndromes. Implications for plaque rupture. Circulation. 1994;90(2):775-8.

30. Tearney GJ, Yabushita H, Houser SL, et al. Quantification of macrophage content in atherosclerotic plaques by optical coherence tomography. Circulation. 2003;107(1):113-9.

31. Tanaka A, Tearney GJ, Bouma BE. Challenges on the frontier of intracoronary imaging: atherosclerotic plaque macrophage measurement by optical coherence tomography. J Biomed Opt. 2010;15(1):011104.

32. Tearney GJ, Jang IK, Bouma BE. Optical coherence tomography for imaging the vulnerable plaque. J Biomed Opt. 2006;11 (2):021002.

33. MacNeill BD, Jang IK, Bouma BE, et al. Focal and multi-focal plaque macrophage distributions in patients with acute and stable presentations of coronary artery disease. J Am Coll Cardiol. 2004;44(5):972-9.

34. Raffel OC, Tearney GJ, Gauthier DD, et al. Relationship between a systemic inflammatory marker, plaque inflammation, and plaque characteristics determined by intravascular optical coherence tomography. Arterioscler Thromb Vasc Biol. 2007;27(8):1820-7.

35. Ferrante G, Nakano M, Prati F, et al. High levels of systemic myeloperoxidase are associated with coronary plaque erosion in patients with acute coronary syndromes: a clinicopathological study. Circulation. 2010;122(24):2505-2513.Study associating systemic elevation in selective inflammatory biomarkers with specific pathologic alterations of the fibrous cap.

36. Garcia-Garcia HM, Costa MA, Serruys PW. Imaging of coronary atherosclerosis: intravascular ultrasound. Eur Heart J. 2010;31 (20):2456-69.

37. Kaneda H, Ako J, Terashima M. Intravascular ultrasound imaging for assessing regression and progression in coronary artery disease. Am J Cardiol. 2010;106(12):1735-46. 
38. Chia S, Raffel OC, Takano M, et al. Association of statin therapy with reduced coronary plaque rupture: an optical coherence tomography study. Coron Artery Dis. 2008;19(4):237-42.

39. • Takarada S, Imanishi T, Kubo T, et al. Effect of statin therapy on coronary fibrous-cap thickness in patients with acute coronary syndrome: assessment by optical coherence tomography study. Atherosclerosis. 2009;202(2):491-497.This study demonstrates the potential of OCT for serially assessing structural changes in the fibrous cap in response to a specific therapy.
40. Ambrose JA. In search of the "vulnerable plaque": can it be localized and will focal regional therapy ever be an option for cardiac prevention? J Am Coll Cardiol. 2008;51(16):1539-42.

41. Stone GW, Maehara A, Lansky AJ, et al. A prospective natural-history study of coronary atherosclerosis. N Engl J Med. 2011;364(3):226-35.

42. Nadkarni SK, Pierce MC, Park BH, et al. Measurement of collagen and smooth muscle cell content in atherosclerotic plaques using polarization-sensitive optical coherence tomography. J Am Coll Cardiol. 2007;49(13):1474-81. 